ISSN 0103-5150

Fisioter. Mov., Curitiba, v. 24, n. 1, p. 39-46, jan./mar. 2011 Licenciado sob uma Licença Creative Commons

\title{
Avaliação da força muscular do assoalho pélvico em idosas com incontinência urinária
}

\author{
Assessment of the pelvic floor muscle strength in elderly female \\ with urinary incontinence
}

\section{Juliana Gonçalves de Sousa ${ }^{[a]}$, Vanessa Ribeiro Ferreira ${ }^{[b]}$, Ricardo Jacó de Oliveira ${ }^{[c]}$, Cláudia Elaine Cestari ${ }^{[\mathrm{d}]}$}

[a] Acadêmica do curso de Fisioterapia da Universidade Católica de Brasília (UCB), Brasília, DF - Brasil, e-mail: jugfisio@gmail.com

[b] Acadêmica do curso de Fisioterapia (UCB), Brasília, DF - Brasil, e-mail: vribeirof@gmail.com

[c] Doutor e professor do Programa de Pós Graduação Stricto Sensu em Educação Física da Universidade Católica de Brasília (UCB), Brasília, DF - Brasil.

[d] Doutora pela Universidade Católica de Brasília, docente do curso de Fisioterapia da Universidade Católica de Brasília (UCB), Brasília, DF - Brasil, e-mail: claudiae@ucb.br

\section{Resumo}

Introdução: A incontinência urinária é considerada um problema de saúde pública e sua prevalência aumenta com o avanço da idade, embora possa acontecer em qualquer fase da vida. São vários os fatores de risco que se associam e contribuem para o aparecimento dos sintomas, entre eles o envelhecimento natural das fibras musculares, a redução da função ovariana após a menopausa, a obesidade, a gravidez, a multiparidade, entre outros. Objetivo: Avaliar a força muscular do assoalho pélvico e a qualidade de vida de mulheres com queixa de incontinência urinária após a cinesioterapia. Materiais e métodos: A amostra foi composta por 22 mulheres submetidas à anamnese, avaliação funcional do assoalho pélvico (AFA), quantificação da contração por meio da palpação bidigital e perineômetro, além da avaliação da qualidade de vida por meio do King's Health Questionnaire (KHQ). As avaliações foram realizadas antes e após a intervenção. 0 protocolo adotado foi composto por exercícios de conscientização e a série de Kegel. Resultados: Em relação ao grau de força muscular foi observada melhora significativa após o tratamento $(p \leq 0,001)$ e melhora do pico de pressão e do tempo de contração mensurados pelo perineômetro ( $\mathrm{p} \leq 0,001)$. Entretanto, não foi observada diferença significativa em relação à resistência muscular e, ao comparar o momento pré e pós-tratamento, 
foi observada melhora na qualidade de vida. No entanto, não foi observada diferença significativa ao se comparar os dois tipos de incontinência em relação à percepção subjetiva de saúde. Conclusão: 0 protocolo cinesioterapêutico adotado foi eficaz para o fortalecimento da musculatura do assoalho pélvico e para a melhora da qualidade de vida em idosas incontinentes.

Palavras-chave: Incontinência urinária. Exercício. Força muscular. Assoalho pélvico. Qualidade de vida.

\section{Abstract}

Introduction: The urinary incontinence is considered a public health problem and the prevalence increases with advancing age, although it can happen at any stage of life. There are several risk factors that are associated and contribute to the beginning of symptoms, among them the natural aging of the muscle fibers, reduction of ovarian function after menopause, obesity, pregnancy, multiparity, among others. To evaluate the pelvic floor muscle strength and quality of life of women complaining of urinary incontinence after kinesiotherapy. Materials and methods: The sample included 22 women whose underwent medical history, functional assessment of the pelvic floor (AFA), quantification of contraction by palpation and bidigital perineometer to the assessment of quality of life through the King's Health Questionnaire (KHQ). Evaluations were performed before and after intervention. The protocol used was composed of awareness exercises and a series of Kegel. Results: In relation to the degree of muscle strength it was observed a significantly improvement after treatment ( $p \leq 0.001)$ improvement in peak pressure and duration of contraction measured at perineometer ( $p \leq 0.001)$. However, there was no significant difference in relation to muscular endurance. Comparing the time before and after treatment, there was improvement in quality of life. However, there was no significant difference when comparing the two types of incontinence in relation to the subjective perception of health. Conclusion: The protocol used was effective kinesiotherapeutic to strengthen the pelvic floor muscles and to improve the quality of life in elderly incontinent.

Keywords: Urinary incontinence. Exercise. Muscle strength. Pelvic floor. Quality of life.

\section{Introdução}

A incontinência urinária (IU) é definida pela Sociedade Internacional de Continência (ICS) como qualquer perda involuntária de urina suficiente para gerar um problema social ou higiênico $(1,2)$. Nesse sentido, a IU é considerada um problema de saúde pública e sua prevalência aumenta com o avanço da idade, embora possa acontecer em qualquer fase da vida (3-5). Alguns fatores de risco podem estar associados ao aparecimento dos sintomas, entre eles o próprio envelhecimento natural das fibras musculares, a redução da função ovariana após a menopausa, a obesidade, a gravidez e os múltiplos partos vaginais (6-8).

De acordo com Grosse e Sengler (9), a prevalência dos sintomas apresentados classifica a IU em:

a) incontinência urinária de urgência (IUU) quando a perda involuntária de urina é acompanhada ou imediatamente precedida de urgência miccional, na presença da hiperatividade do músculo detrusor (6); b) incontinência urinária de esforço (IUE) - quando a pressão vesical excede a pressão máxima de fechamento uretral, na ausência de contração do detrusor (10-14); e

c) incontinência urinária mista (IUM) - quando ocorre a associação dos sintomas anteriormente citados (6).

A IUE é o tipo mais comum de perda involuntária de urina na mulher, podendo ocorrer por hipermobilidade da uretra ou por deficiência esfincteriana, sendo a hipermobilidade a causa mais frequente, podendo ocorrer em decorrência da fraqueza do assoalho pélvico ou por consequência de procedimentos cirúrgicos $(12,13)$, já a deficiência esfincteriana intrínseca é causada pela incapacidade do esfíncter uretral em manter a coaptação da mucosa, tanto no repouso quanto no exercício $(15,16)$.

Independente do tipo de IU, os prejuízos para a qualidade de vida são inúmeros e, por isso, a ICS tem recomendado a aplicação de um questionário para melhor avaliar esse problema (17). Existem vários 
questionários que podem ser utilizados em mulheres incontinentes com esse objetivo, podendo ter um caráter genérico ou específico. Dentre os específicos destaca-se o King's Health (KHQ), por investigar tanto a presença de sintomas de IU quanto seu impacto relativo, levando a resultados mais consistentes $(17,18)$.

Para a adesão da paciente ao tratamento e o sucesso da intervenção terapêutica é importante observar como cada paciente percebe a IU e as consequências trazidas pela patologia para sua vida, de forma que, mesmo apresentando sinais e sintomas semelhantes, possam adotar diferentes estratégias para enfrentar o problema $(6,19)$. Dentre as estratégias, destacam-se as intervenções cirúrgicas e o uso de medicamentos, além das terapias conservadoras.

0 tratamento cirúrgico, além de ser um procedimento invasivo, pode trazer complicações, apresentar elevado custo e altas recidivas $(6,14,20)$. A terapia medicamentosa uma vez iniciada não deve ser interrompida e ainda pode causar efeitos colaterais (6). Por esses motivos, vem aumentando o interesse por tratamentos mais conservadores como a fisioterapia (14). Sendo assim, a ICS, em 2005, recomendou o tratamento fisioterapêutico para IU como a primeira opção, pois se trata de técnicas e recursos que envolvem baixo custo e risco, além de eficácia comprovada (6). Entre as modalidades desse tratamento destacam-se a eletroestimulação, o biofeedback e a cinesioterapia. A cinesioterapia visa essencialmente ao fortalecimento muscular, estratégia tão imprescindível quanto avaliar a capacidade que as mulheres têm em contrair corretamente esse grupo muscular (21-24). Dessa forma, a cinesioterapia é uma técnica que permite, por meio da contração e do relaxamento dos músculos do assoalho pélvico, resultados mais efetivos, observando-se melhora ou cura em vários pacientes de forma mais duradoura (25-27).

Nesse sentido, o propósito desse estudo foi verificar se após a cinesioterapia houve melhora da força da musculatura do assoalho pélvico e da qualidade de vida em mulheres pós-menopáusicas com queixa clínica de incontinência urinária de esforço ou mista.

\section{Materiais e métodos}

O estudo, do tipo experimental, foi desenvolvido no Setor de Fisioterapia Uroginecológica e Obstétrica do Hospital da Universidade Católica de Brasília, no período de setembro de 2007 a setembro de 2008. A amostra foi composta inicialmente por 25 mulheres pós-menopáusicas. Destas, somente 22 concluíram o estudo, pois uma paciente apresentou restrição anatômica, impossibilitando a realização do exame, e duas desistiram por motivos pessoais.

Todas as pacientes assinaram o termo de consentimento livre e esclarecido antes de iniciar o estudo, mediante aprovação pelo Comitê de Ética em Pesquisa da Instituição, registrado pelo número CEP/UCB 014/2007. Foram incluídas mulheres que referiram queixa clínica de perda urinária aos esforços e urgência miccional e que cumpriram adequadamente os protocolos propostos e não apresentaram infecção urinária no período de intervenção e nem restrições anatômicas durante o exame.

As pacientes foram submetidas a uma anamnese acerca dos sintomas urinários, índice de massa corpórea (IMC), número de gestações, número e tipo de partos, presença ou ausência de menopausa, uso de reposição hormonal, presença de infecções urinárias, sensações de urgência, perda de urina aos esforços, além da pesquisa de doenças associadas, tais como diabetes mellitus (DM) e hipertensão arterial sistêmica (HAS).

A avaliação funcional da musculatura do assoalho pélvico (AFA) foi realizada pela palpação bidigital e pela quantificação da contração perineal, utilizando o perineômetro. 0 exame da palpação bidigital foi realizado com a paciente em decúbito dorsal em posição ginecológica modificada (flexoabdução de coxofemoral com os pés apoiados sobre a maca); os dedos indicador e médio do examinador foram introduzidos no canal vaginal, com a mão devidamente enluvada e untada em gel. Foi solicitado à paciente que contraísse a musculatura ao redor dos dedos do examinador e sustentasse essa contração pelo tempo máximo que conseguisse. Para avaliação da força de contração e resistência da musculatura do assoalho pélvico foi utilizada a classificação de Ortis et al. (28).

Para avaliar a pressão de contração exercida pela musculatura do assoalho pélvico foi utilizado o perineômetro, tipo Perina 996-2, marca Quark. Tal aparelho atende a todos os requisitos da norma de segurança para equipamentos eletromédicos IEC 601 (norma geral) e IEC 601-2-10 (norma particular para eletroestimuladores). A sonda intracavitária, conectada a um manômetro de pressão e protegida por um preservativo não lubrificado, 
foi untada em gel e introduzida no canal vaginal. A válvula foi fechada e, na sequência, a sonda foi insuflada lentamente até que a paciente sentisse o contato da sonda com a parede vaginal, sem referir dor, mas levando a uma ligeira distensão da parede vaginal. Após o ajuste do aparelho, a resistência foi modulada e foi solicitado à paciente que contraísse a musculatura do assoalho pélvico por três vezes consecutivas e mantivesse a contração pelo tempo máximo que conseguisse. Foram observados o pico de pressão, o tempo e a resistência que os músculos perineais permaneceram contraídos. As médias das medidas realizadas pelo perineômetro foram consideradas para análise. No final da aferição, a sonda foi desinflada abrindo-se a válvula e depois foi retirada.

Para a avaliação da qualidade de vida foi utilizado o questionário King's Health (KHQ), o qual foi aplicado de forma assistida pelos pesquisadores ao início e ao término do protocolo fisioterapêutico escolhido. $\mathrm{O}$ KHQ é composto por trinta perguntas que são distribuídas em nove domínios: percepção da saúde, impacto da incontinência, limitações do desempenho das tarefas, limitação física, limitação social, relacionamento pessoal, emoções, sono, disposição e medidas de gravidade (17).

A todas as respostas foram atribuídos valores numéricos, somados e avaliados por domínio. Os valores foram então calculados por meio de fórmula matemática, obtendo-se assim o escore de qualidade de vida, que varia de 0 a 100 , considerando-se que quanto maior o número obtido pior a qualidade de vida (17).

0 tratamento cinesioterapêutico utilizado foi composto por exercícios de conscientização e da série de Kegel, sendo realizados duas vezes por semana, de forma individualizada, com duração média de 30 minutos para cada paciente e totalizando 12 sessões de atendimento.

Durante a fase de conscientização foi solicitado à paciente que realizasse contrações lentas e submáximas intercaladas com relaxamento, já que se trata de uma musculatura extremamente fatigável. Durante essa fase, a paciente permaneceu na posição ginecológica modificada e o terapeuta, após localizar a tuberosidade isquiática com os seus polegares, medializou-a, palpando o núcleo fibroso central do períneo. Esse exercício foi composto por cinco etapas, e cada etapa foi repetida quinze vezes consecutivas: a primeira consistiu em "apertar", exercendo uma leve pressão na região; na segunda, a pressão veio seguida de tração em direção caudal; na terceira, além da pressão e tração, foi solicitado que a paciente executasse uma contração voluntária, acompanhada de forma assistida pelo examinador. Na quarta, todos os passos anteriores foram acompanhados de uma resistência opositora parcial do examinador durante toda a contração; e na última etapa, após as fases anteriores, a contração voluntária da paciente foi exercida contra a resistência do examinador e ao final foi realizado um reflexo de estiramento.

Por sua vez, a série de Kegel baseou-se em contrações voluntárias da musculatura do assoalho pélvico. Como não existe consenso na literatura quanto à padronização dessa técnica (4), optamos pela realização do seguinte protocolo: quatro contrações lentas com duração de cinco segundos e intervalo de dez segundos entre cada contração e, em seguida, oito contrações rápidas sem relaxamento entre elas. Esse conjunto de contrações correspondeu a uma série e o protocolo adotado consistiu em três séries de Kegel. A série de Kegel intercala as contrações mantidas com as rápidas com o propósito de estimular as fibras musculares do tipo I e do tipo II do assoalho pélvico (20).

Ao final do tratamento cinesioterapêutico foi realizada a reavaliação composta pela AFA, pelo perineômetro e a aplicação do KHQ. Para a análise estatística foram utilizados a média e o desvio-padrão, por meio da distribuição da frequência, a fim de caracterizar as variáveis clínicas. Para verificar o grau de diferença sistemática da AFA e do perineômetro antes e após o tratamento foi utilizado o teste $t$ pareado e, após os cálculos para se obter os escores do questionário de $\mathrm{QV}$, foram utilizados também a média e o desvio-padrão comparando-se os momentos pré e pós-intervenção. Os dados foram tratados no software SPSS, versão 16.0. Para efeito de análise foi adotado um nível de significância de $\mathrm{p} \leq 0,05$.

\section{Resultados}

Os resultados referentes às características descritivas da amostra das 22 pacientes avaliadas e tratadas estão demonstrados na Tabela 1.

Em relação ao tipo de queixa urinária, observouse que das 22 mulheres estudadas 14 apresentaram queixa clínica de IUE $(63,64 \%)$ e 8 de IUM $(36,36 \%)$. 
Tabela 1 - Médias e desvios-padrão das características descritivas das 22 mulheres com queixa clínica de incontinência urinária

\begin{tabular}{lccc}
\hline Variáveis & \multicolumn{1}{c}{$\mathrm{X} \pm \mathrm{DP}$} & Mínimo & Máximo \\
\hline Idade (anos) & $65,644,09$ & 65 & 74 \\
IMC & $27,72 \pm 3,25$ & 22,35 & 33,2 \\
Partos & $5,53 \pm 2,79$ & 0 & 13 \\
Gestações & $6,59 \pm 2,59$ & 4 & 14 \\
\hline
\end{tabular}

Legenda: $X$ = média; DP = desvio-padrão; IMC = índice de massa corporal.

Apenas três pacientes $(13,64 \%)$ realizaram terapia com reposição hormonal - TRH. Quanto às doenças associadas, duas apresentaram DM $(9,09 \%)$ e onze HAS (50\%).

Na Tabela 2, está demonstrado que houve aumento significativo no grau de força muscular após o tratamento $(\mathrm{p} \leq 0,05)$ e aumento no pico e no tempo de contração. No entanto, não foi encontrada diferença significativa em relação à resistência de contração, pois ela se manteve igual antes e após o tratamento (Tabela 2).

Considerando-se os dados referentes à média e ao desvio-padrão, mínimos e máximos, obtidos nos domínios do KHQ, foi observada diminuição dos escores do questionário ao se realizar uma comparação do momento pré e pós-tratamento, verificando-se melhora na QV após o tratamento fisioterapêutico em todos os domínios do questionário (Tabela 3).

Ao se realizar a correlação dos domínios do KHQ com a queixa clínica apresentada pelas pacientes, se IUE ou IUM, não foi observada nenhuma diferença estatisticamente significativa $(p>0,05)$ em relação à percepção subjetiva de saúde (Tabela 4).

\section{Discussão}

A amostra escolhida foi composta de mulheres com faixa etária bem avançada, pois, segundo Lopes et al. (3) e Higa et al. (7), a incidência da IU aumenta com a idade e é mais frequente no sexo feminino. No presente estudo houve uma prevalência de IUE, concordando com Figueiredo et al. (6) e Lopes et al. (3). No entanto, Figueiredo et al. (6) afirmaram que a segunda forma de IU mais frequente é a mista seguida
Tabela 2 - Médias e desvios-padrão da avaliação funcional do assoalho pélvico (AFA) e da pressão de contração obtida pelo perineômetro

\begin{tabular}{lcc}
\hline Variáveis & $\begin{array}{c}\mathrm{X} \pm \mathrm{DP} \\
\text { Pré }\end{array}$ & $\begin{array}{c}\mathrm{X} \pm \mathrm{DP} \\
\text { Pós }\end{array}$ \\
\hline AFA & $1,35 \pm 0,92$ & $3,78 \pm 0,51^{*}$ \\
Pico (unidade) & $0,44 \pm 0,30$ & $1,0 \pm 0,44^{*}$ \\
Resistência & & \\
Tempo (unidade) & $1,65 \pm 1,15$ & $4,43 \pm 1,03^{*}$ \\
\hline
\end{tabular}

Legenda: * $=$ resultado significativo entre 0 pré e pós-tratamento $(p \leq 0,001)$.

Tabela 3 - Características descritivas dos escores nos domínios do KHQ

\begin{tabular}{lcc}
\hline Domínios do KHQ & Pré & Pós \\
\hline PGS & $37,9 \pm 23,7$ & $27,3 \pm 21,7$ \\
$\|$ & $27,3 \pm 18,6$ & $0,1 \pm 0,1$ \\
LAD & $25,8 \pm 31,2$ & $20,5 \pm 32,4$ \\
LF & $28,0 \pm 25,4$ & $14,0 \pm 23,9$ \\
LS & $14,4 \pm 25,4$ & $4,5 \pm 21,3$ \\
RP & $26,1 \pm 25,6$ & $17,4 \pm 23,3$ \\
E & $35,4 \pm 31,2$ & $23,7 \pm 28,4$ \\
S/D & $23,5 \pm 21,6$ & $12,9 \pm 16,2$ \\
MG & $42,1 \pm 16,1$ & $30,3 \pm 17,1$ \\
\hline Total & $28,9 \pm 24,3$ & $16,8 \pm 20,5$ \\
\hline
\end{tabular}

Legenda: $X$ = média; DP = desvio-padrão; PGS = percepção geral da saúde; $\|$ = impacto da incontinência; LAD = limitações de atividades diárias; $L F=$ limitações físicas; $L S=$ limitações sociais; $\mathrm{RP}=$ relações pessoais; $\mathrm{E}=$ emoções; $\mathrm{S} / \mathrm{D}=$ sono e disposição; $\mathrm{MG}=$ medidas de gravidade.

da urge-incontinência, discordando de Lopes et al. (3), que afirmaram o inverso.

Neste estudo, a população estudada, apesar de não estar acima do peso, não apresentava obesidade, o que pode ser constatado por meio do IMC. Higa et al. (7) relataram que uma alteração anatômica pela sobrecarga de peso no assoalho pélvico é um fator predisponente para IU. Nesse sentido, o número elevado de gravidez e de partos vaginais verificados na amostra selecionada pode ter sido uma causa importante e contribuído para desenvolver IU $(6,7)$. 
Tabela 4 - Correlação nos domínios do KHQ de acordo com a queixa clínica apresentada

\begin{tabular}{lrcccc}
\hline & \multicolumn{2}{c}{ IUM } & & \multicolumn{2}{c}{ IUE } \\
\cline { 2 - 3 } \cline { 5 - 6 } Domínios do KHQ & \multicolumn{1}{c}{ Pré } & \multicolumn{1}{c}{ Pós } & & Pré & Pós \\
\hline PGS & $45,8 \pm 30,5$ & $34,3 \pm 29,6$ & & $33,3 \pm 18$, & $23,2 \pm 15,3$ \\
II & $22,7 \pm 21,5$ & $13,4 \pm 15,3$ & & $29,8 \pm 16,9$ & $13,5 \pm 13,4$ \\
LAD & $25 \pm 38,8$ & $28,1 \pm 41$ & & $26,1 \pm 27,5$ & $16 \pm 27$ \\
LF & $37,5 \pm 29,2$ & $18,7 \pm 33,8$ & & $22,6 \pm 22,2$ & $11,3 \pm 16,8$ \\
LS & $23,9 \pm 34,9$ & $12,5 \pm 35,3$ & & $8,9 \pm 17,1$ & 0 \\
RP & $34,7 \pm 30,8$ & $29,1 \pm 31,8$ & & $21,2 \pm 21,8$ & $10,7 \pm 14$ \\
E & $43 \pm 42,5$ & $36,8 \pm 33,5$ & & $30,9 \pm 23,1$ & $16,2 \pm 23$ \\
S/D & $16,6 \pm 15,4$ & $14,5 \pm 18,7$ & & $27,3 \pm 24,1$ & $11,9 \pm 15,2$ \\
MG & $46,6 \pm 20,1$ & $37 \pm 21,5$ & & $39,5 \pm 13,4$ & $26,4 \pm 13,2$ \\
\hline
\end{tabular}

Legenda: PGS = percepção geral da saúde; II = impacto da incontinência; LAD = limitações de atividades diárias; LF = limitações físicas; $L S=$ limitações sociais; $R P=$ relações pessoais; $E=$ emoções; $S / D=$ sono e disposição; $M G=$ medidas de gravidade.

Somente três pacientes estudadas fizeram uso de terapia de reposição hormonal. Esse dado é importante, pois o hipoestrogenismo após a menopausa prejudica a coaptação da mucosa da uretra, fato esse que foi explicado por Figueiredo et al. (6).

A existência de HAS e DM foi observada em 11 e 2 pacientes respectivamente, o que, de acordo com Rett et al. (14), pode agravar ou causar IU, representando dois dos principais fatores de risco para a ocorrência desse distúrbio. Com frequência, essas doenças levam à invalidez parcial ou total do indivíduo, porém, quando diagnosticadas precocemente, são bastante sensíveis, oferecendo múltiplas chances de evitar complicações, retardando a progressão das doenças já existentes e as perdas resultantes de tais doenças (14).

De acordo com os resultados obtidos, a cinesioterapia é uma das formas de tratamento mais eficazes para o fortalecimento dos músculos do assoalho pélvico, podendo ser aplicada isoladamente ou associada a outras técnicas $(4,20)$. 0 presente estudo demonstrou que a força de contração da musculatura pélvica aumentou significativamente com os exercícios de Kegel após seis semanas de tratamento, concordando com Yoon et al. (29).

Esse aumento da força muscular pode ser observado por meio dos resultados das medidas avaliativas objetivas AFA e perineômetro, que demonstraram valores estatisticamente maiores após a intervenção cinesioterapêutica, se comparados ao momento anterior a essa intervenção, o que pode ser também observado no estudo de Figueiredo et al. (6) e de Bernardes et al. (20), indicando que as pacientes apresentaram baixas percepções corporais e fraca capacidade de contração da musculatura pélvica antes da intervenção.

Moreira et al. (12) observaram uma média inferior quando realizaram a avaliação funcional do assoalho pélvico em mulheres incontinentes na pós-menopausa do que em mulheres continentes, mostrando que as incontinentes apresentavam fraqueza do assoalho pélvico maior do que mulheres sem perda urinária, ressaltando a importância de protocolos cinesioterapêuticos que fortaleçam essa musculatura.

As pacientes estudadas realizaram os exercícios sob a supervisão de um fisioterapeuta para orientação dos comandos, o que pode ser encontrado também no trabalho de Zanetti et al. (4), em que 30\% das mulheres foram incapazes de contrair corretamente a musculatura pélvica e apresentavam alta incidência de abandono quando não orientadas por um profissional.

Bernardes et al. (20) concordam com os autores anteriormente citados, assegurando, ainda, que é de responsabilidade do fisioterapeuta orientar e motivar as pacientes quanto à realização dos exercícios em casa, graças a altas recidivas da IU após cinco anos sem manutenção dessa terapia.

O questionário adotado no estudo - KHQ - foi importante para avaliar a $\mathrm{QV}$, sendo este de alta confia- 
bilidade e validade, mostrando ser um instrumento adequado para pacientes incontinentes $(14,17,30)$. É imprescindível avaliar a QV, como afirma Rett et al. (14), levando-se em consideração o impacto que a IU causa na vida das pacientes.

Nos resultados apresentados, assim como nos estudos de Zanetti et al. (4) e de Rett et al. (14), a QV melhorou após a intervenção cinesioterapêutica. No entanto, não foi detectada nenhuma correlação significativa ao se comparar os dois tipos de IU com a percepção subjetiva de saúde, diferindo do estudo de Rett et al. (14), em que a IUU e a IUM causaram maior prejuízo na QV do que a IUE. Isso pode ser explicado em parte pela maneira com que cada paciente percebe seus sintomas, bem como sua gravidade e o contexto cultural no qual estão inseridas $(6,19)$.

\section{Conclusão}

Este estudo permitiu concluir que o tratamento cinesioterapêutico fortaleceu a musculatura do assoalho pélvico e contribuiu para a melhora da qualidade de vida em mulheres pós-menopáusicas com queixa clínica de incontinência urinária de esforço ou mista.

\section{Referências}

1. Abrams P, Blaivas JG, Stanton SL, Andersen JT. The standardization of terminology of lower urinary tract function. Scand J Urol Nephrol. 1988;114:5-19.

2. Kluber L, Moriguche EH, Cruz IBM. A influência da fisioterapia na qualidade de vida em mulheres com incontinência urinária: revisão. Rev Med PUCRS. 2002; 12(3):243-9.

3. Lopes MHBM, Higa R. Restrições causadas pela incontinência urinária à vida da mulher. Rev Esc Enferm. 2006;40(1):39-41.

4. Zanetti MRD, Castro RA, Rotta AL, Santos PD, Sartori M, Girão MJBC. Impact of supervised physiotherapeutic pelvic floor exercises for treating female stress urinary incontinence. São Paulo Med J. 2007;125(5):265-9.

5. Luft J, Uriheas-Nichols AA, Identifying the risk factors for developing incontinence, can we modify individual risk. Geriatr Nurs. 1998;(19):66-71.
6. Figueiredo EM, Lara JO, Cruz MC, Quintão DMG, Monteiro MVC. Perfil sociodemográfico e clínico de usuárias de serviço de fisioterapia uroginecológica da rede pública. Rev Bras Fisioter. 2008;12(2): 136-42.

7. Higa R, Lopes MHBM, Reis MJ. Factores de riesgo para incontinencia urinaria en la mujer. Rev Esc Enferm. 2008;42(1):187-192.

8. Hill GMD. Diferenças culturais em relação ao envelhecimento. In: Pickles B. Fisioterapia na terceira idade. São Paulo: Atheneu; 2000. p. 43-53.

9. Grosse D, Sengler J. Reeducação perineal: concepção, realização e transcrição em prática liberal e hospitalar. São Paulo: Manole; 2002.

10. de Lancey YOL, Ashton-Miller JA. Pathophysiology of adult urinary incontinence. Gastroenterology. 2004; 126(1):523-32.

11. Rubstein I, Rubstein M. Avaliação diagnóstica e classificação da incontinência urinária de esforço. In: Rubstein I, editor. Urologia feminina. São Paulo: BYK; 1999. p. 179-88.

12. Moreira SFS, Girão MJBC, Sartori MGF, Baracat EC, Lima GR. Mobilidade do colo vesical e avaliação funcional do assoalho pélvico em mulheres continentes e com incontinência urinária de esforço, consoante o estado hormonal. Rev Bras Ginecol Obstet. 2002; 24(6):365-70.

13. Feldner Jr PC, Bezerra LRPS, Girão MJBC, Castro RA, Sartori MGF, Baracat EC, et al. Correlação entre a pressão de perda à manobra de valsalva e a pressão máxima de fechamento uretral com a história clínica em mulheres com incontinência urinária de esforço. Rev Bras Ginecol Obstet. 2002;24(2):87-91.

14. Rett MT, Simões JA, Herrmann V, Gurgel MSC, Morais SS. Qualidade de vida em mulheres após tratamento da incontinência urinária de esforço com fisioterapia. Rev Bras Ginecol Obstet. 2007;29(3):134-0.

15. Fernandes CE, Morita MH, Ferreira JAS, Silva EP, Wehba S. Abordagem dos distúrbios do trato urinário na mulher pós-menopausada. Rev Paul Med. 1990; 108:230-5.

16. Feldner Jr PC, Sartori MGF, Lima GR, Baracat EC, Girão MJBC. Diagnóstico clínico e subsidiário da incontinência urinária. Rev Bras Ginecol Obstet. 2006; 28(1):54-62. 
17. Fonseca ESM, Camargo ALM, Castro RA, Sartori MGF, Fonseca MCM, Lima GR, et al. Validação do questionário de qualidade de vida (King's Health Questionnaire) em mulheres brasileiras com incontinência urinária. Rev Bras Ginecol Obstet. 2005; 27(5):235-42.

18. Huskaar S, Vinsnes A. The quality of life in women with urinary incontinence as measured by the sickness impact profile. J Am Geriatr Soc. 1991;39:378-82.

19. Abreu NS, Baracho ES, Tirado MGA, Dias RC. Qualidade de vida na perspectiva de idosas com incontinência urinária. Rev Bras Fisioter. 2007;11(6):429-36.

20. Bernardes NO, Peres FR, Souza ELBL, Souza OL. Métodos de tratamento utilizados na incontinência urinária de esforço genuína: um estudo comparativo entre cinesioterapia e eletroestimulação endovaginal. Rev Bras Ginecol Obstet. 2000;22(1):49-54.

21. de Lancey JO. Structural aspect uretrovesical function in the female. Neurourol. 1980;(7):509-19.

22. Mourin M, Dumoulin C, Bourbonnais D. Pelvic floor maximal strength using vaginal digital assessment compared to dynamometric measurements. Neurourol Urody. 2004; 23(4):336-41.

23. Dumoulin C. Efficanté des traitements pysiotherapiques pour I'incontinence urinaire d'effort chez la femme em période postnatale [tese]. Otawa: Bibliothèque et Archives Canadá; 2003.

24. Moreno AL. Fisioterapia em uroginecologia. Barueri: Manole; 2004.
25. Bo K, Maanum M. Does vaginal electrical stimulation cause pelvic floor muscle contraction? A pilot study. Scand J Urol Nephrol. 1996;179(Suppl.):39-45.

26. Morkved S, Kari BO, Toril F. Effect of adding biofeedback to pelvic floor muscle training to treat urodynamic stress incontinence. Rev Bras Ginecol Obstet. 2002;100(4):730-9.

27. Hay-Smith EJ, Dumoulin C. Pelvic floor muscle training versus no treatment, or inactive control treatment, for urinary incontinence in women. Cochrane Database Syst Rev. 2006;(1):CD005654.

28. Ortiz OC, Coya NF, Ibanez G. Evaluación funcional del piso pelviano femenino (clasificación funcional). Soc Latinoam Uroginecol Cir Vaginal. 1996;1:5-9.

29. Yoon HS, Song HH, Ro YJ. A comparison of effectiveness of bladder training and pelvic muscle exercise on female urinary incontinence. Inter J Nursing Studies. 2003;40(1):45-50.

30. Tamanini JTN, D'Ancona CAL, Botega NJ, Netto Jr NR. Validação do "King's Health Questionnaire" para o português em mulheres com incontinência urinária. Rev Saúde Pública. 2003;37(2):203-11.

Recebido: 23/04/2010 Received: 04/23/2010

Aprovado: 04/11/2010 Approved: 11/04/2010 